\title{
Application of a Novel Large-Volume Injection Method Using a Stomach-Shaped Inlet Liner in Capillary Gas Chromatographic Trace Analysis of Dioxins in Human Milk and Plasma
}

\author{
Koichi Saito, ${ }^{* 1 \dagger}$ Atsuko Ohmura, ${ }^{* 2}$ Mikiko Takekuma, ${ }^{* 2}$ Ryoichi Sasano, ${ }^{* 3}$ Yasuhiko Matsuki, $* 4$ \\ and Hiroyuki NAKAZAWA*1 \\ *1 Department of Analytical Chemistry, Faculty of Pharmaceutical Sciences, Hoshi University, \\ 2-4-41 Ebara, Shinagawa, Tokyo 142-8501, Japan \\ *2 Biological Effect Research Group, Saitama Institute of Public Health, \\ 639-1 Kamiokubo, Sakura, Saitama 338-0824, Japan \\ *3 AiSTI SCIENCE Co., Ltd., 75-2 Kuroda, Wakayama 640-8341, Japan \\ *4 Institute of Food Hygiene, Japan Food Hygiene Association, 2-5-47 Tadao, Machida, Tokyo 194-0035, Japan
}

\begin{abstract}
A newly developed large-volume injection (LVI) technique that employs a unique stomach-shaped inlet liner (SSIL) inside of a programmable temperature vaporizer was used for the determination of trace amounts of dioxins in human milk and plasma. The initial temperature and the initial dwelling time of the inlet and the kind of solvent used were found to be critical in determining the analytical sensitivity of dioxins due to the loss of these relatively volatile compounds during solvent vaporization. Human milk and plasma were purified and fractionated by pre-packed multi-layered silicagel chromatography and activated carbon silica-gel column chromatography. A $20-\mu \mathrm{L}$ aliquot of the fraction collected from the chromatography with toluene was directly applied to the LVI system in high-resolution gas chromatography/high-resolution mass spectrometry. Excellent correlation $(r>0.97)$ between the values obtained by the LVI method using the SSIL device and those by the conventional regular-volume splitless injection method was obtained for PCDDs, PCDFs and non-ortho PCBs in human milk and plasma samples.
\end{abstract}

(Received March 13, 2007; Accepted May 7, 2007; Published June 10, 2007)

\section{Introduction}

The contamination of food and the ecosystem by dioxins and the resultant effects on human health have been drawing much attention from the general public. In this regard, the investigation of human exposure to dioxins is an urgent task that needs to be undertaken by the Japanese government. Since "The Law Concerning Special Measures against Dioxins" took effect in Japan in 1999, the number of substances to be sampled and measured has been increasing. An official analytical method for dioxins in human milk, blood and food was established in Japan. However, there remain some problems concerning the analytical method, one of which is a need to concentrate the dioxin fraction by solvent evaporation by a nitrogen gas stream at the final stage before high-resolution gas chromatography/high-resolution mass spectrometry (HRGC/HRMS). The conventional solvent evaporation by a nitrogen gas stream is cumbersome, time consuming, and needs carefully controlled operation by an expert. A loss of dioxin may be caused by accompanying solvent vaporization, when a less-experienced laboratory technician carries out the solvent evaporation. It thereby results in a lowering of the reliability of the experimental data. As for other problems, solvent

$\dagger$ To whom correspondence should be addressed.

E-mail: k-saito@hoshi.ac.jp evaporation by a nitrogen gas stream has the possibility of causing the exposure to dioxins of laboratory technicians who perform the operation. In order to enhance the detection sensitivity without any concentration of the solvent by a nitrogen gas stream, as described above, several large-volume injection (LVI) techniques have been proposed $^{1,2}$ for a gas chromatography/mass spectrometry (GC/MS). Also, several applications of the LVI techniques for the analysis of pesticides have been reported; ${ }^{3-7}$ however, there are few applications to dioxin analysis. ${ }^{8-11}$

The representative LVI methods were a programmable temperature vaporizing (PTV) injection method ${ }^{12-14}$ and a largevolume on-column injection method. ${ }^{15,16}$ Also, other available methods were loop-type, ${ }^{17,18}$ vapor-overflow ${ }^{19,20}$ and concurrent solvent recondensation $(\mathrm{CSR})^{21}$ techniques. In the conventional PTV injection method, the sample is evaporated in the inlet, and during sample introduction the solvent can be eliminated via a split outlet, while higher boiling-point analytes are retained and subsequently transferred to the separating column in the splitless mode. One of the conventional PTV injection methods needs a liner packed with materials, such as silica wool, ${ }^{10}$ Chromosorb-based material, ${ }^{14}$ Tenax adsorbent ${ }^{22}$ and phenylmethylsilicone chemically bonded silica, ${ }^{23}$ which are adequate to retain the target solutes while the solvent is simultaneously removed through the split vent line. The other type of PTV is the so-called At-column concentrating method, ${ }^{24}$ in which a small-diameter hole is found at the side of an inlet 
liner, which serves as a vent for the evaporated solvent. The Atcolumn concentrating method combines features of two other injection techniques: loop-type large-volume and vapor overflow methods. However, these conventional PTV methods have drawbacks, i.e., since the conventional PTV injection method that uses a straight inlet liner, usually packed with the materials described above, some analytes adsorb to the surface of materials where they likely decompose due to the catalytic effect of the materials. ${ }^{25,26}$ As for the At-column concentrating method, strict temperature control between the injection port and the column oven is required, and the temperature setting of the GC oven is crucial. Furthermore, high boiling-point solvents, such as toluene, nonane and decane, cannot be used because a large difference in the boiling-point between the solvent and the analyte is needed. In addition, the volume of one injection is limited because the capacity of the straight inlet liner is small in the At-column concentrating method.

On the other hand, in the large-volume on-column injection method, a precolumn is employed to separate the solvent and the analytes of interest. In the system, the large-volume sample solution is directly injected onto a precolumn, and then the solvent is excreted via a solvent vapor exit installed between the precolumn and an analytical column. Although it gained some popularity at the beginning, the on-column injection method is now largely replaced with the popular PTV methods, because it requires an extremely complicated GC system, such as a precolumn, a cold trap system for sample concentration, and valves for carrier gas switching. Furthermore, the injection speed of the autosampler should be carefully controlled to prevent flooding of the solvent. ${ }^{27,28}$ In addition, a loss of volatile analytes and carry-over due to liquid left behind in the transfer capillary located between a valve and the column may occur.

In order to overcome the drawbacks of the injection techniques described above, a modified PTV method using a unique stomach-shaped inlet liner (SSIL) in the injection port has been developed as a novel LVI technique. In this paper, we have applied and evaluated the SSIL injection technique for the determination of trace amounts of dioxins in human milk and plasma.

\section{Experimental}

\section{Materials and chemicals}

All dioxin standards were from Wellington Laboratories (Canada) and were diluted with decane to the appropriate concentrations. Most of the organic solvents, including hexane, acetone, dichloromethane, toluene, diethyl ether, ethanol and methanol $(\mathrm{MeOH})$, were of dioxin analysis grade and were from Kanto Kagaku (Tokyo, Japan) or Wako Pure Chemical Industries (Osaka, Japan). Decane was of special grade and was redistilled prior to use. All other chemicals were of PCB analysis grade or special grade. The multi-layered silica-gel column packed in a disposable cartridge tube was from Supelco (USA). It consisted of the following layers: $0.9 \mathrm{~g}$ of silica-gel, $3 \mathrm{~g}$ of $2 \% \mathrm{KOH} /$ silica-gel, $0.9 \mathrm{~g}$ of silica-gel, $4.5 \mathrm{~g}$ of $44 \%$ $\mathrm{H}_{2} \mathrm{SO}_{4} /$ silica-gel, $6 \mathrm{~g}$ of $22 \% \mathrm{H}_{2} \mathrm{SO}_{4} /$ silica-gel, $0.9 \mathrm{~g}$ of silica-gel and $3 \mathrm{~g}$ of $10 \% \mathrm{AgNO}_{3} /$ silica-gel. Six grams of sodium sulfate were manually added on top of the $\mathrm{AgNO}_{3} /$ silica-gel layer in the column. The column was washed with $100 \mathrm{~mL}$ of hexane prior to use.

\section{Analysis of dioxins}

In this experiment, 7 kinds of polychlorinated dibenzo- $p$ - dioxins (PCDDs), 10 kinds of polychlorinated dibenzofurans (PCDFs), 4 kinds of non-ortho PCBs and 8 kinds of mono-ortho PCBs, all of which are defined in WHO-TEF (1998), were analyzed as dioxins. Human milk samples were collected from 17 Japanese primiparae, and plasma samples were collected from 4 volunteers. A sample pretreatment for dioxin analysis was carried out in accordance with a slightly modified method from the official manual of the Ministry of Health, Labour and Welfare, Japan. Briefly, approximately $50 \mathrm{~g}$ of a milk (or plasma) sample was used for the analysis. Fat was extracted from the sample according to a previously described procedure. ${ }^{29}$ Each stable isotope labeled congener of PCDD/Fs, non-ortho and mono-ortho PCBs was added as a surrogate. The fat was dissolved in ca. $2 \mathrm{~mL}$ of hexane and applied to a multilayered silica-gel column. After eluting the column with 160 $\mathrm{mL}$ of hexane, the eluate was concentrated to $c a .1 \mathrm{~mL}$. This was subjected to chromatography on an activated carbon/silicagel column $(0.5 \mathrm{~g}$ of activated carbon/silica-gel was pre-packed in a manner similar to that described previously), ${ }^{30}$ and was subsequently fractionated as follows. First, $10 \mathrm{~mL}$ of hexane was added to elute most of the non-planar PCBs, and then 40 $\mathrm{mL}$ of $25 \%$ dichloromethane/hexane was added to elute 8 mono-ortho PCBs. Finally, $100 \mathrm{~mL}$ of toluene was added to elute $17 \mathrm{PCDD} / \mathrm{Fs}$ and 4 non-ortho $\mathrm{PCBs}$. The toluene fraction was concentrated to $2 \mathrm{~mL}$, and then divided equally into two portions. One was evaporated to near dryness with a rotary evaporator and a solvent evaporation by nitrogen gas stream. Then, $50 \mu \mathrm{L}(10 \mu \mathrm{L}$, in the case of plasma sample) of an internal standard decane solution for calibration was added to reconstitute the sample. One microliter aliquot was injected by the conventional splitless injection for the determination of PCDD/Fs and non-ortho PCBs, respectively. On the other hand, to the remaining portion $(1 \mathrm{~mL})$ of the toluene fraction (concentrated to $c a .200 \mu \mathrm{L}$ in the case of plasma sample) was added $50 \mu \mathrm{L}(10 \mu \mathrm{L}$, in the case of plasma sample) of an internal standard decane solution for calibration, and $20 \mu \mathrm{L}$ aliquots of the solution were subjected to the LVI method for the determination of PCDD/Fs and non-ortho PCBs, respectively. Mono-ortho $\mathrm{PCBs}$ were determined by the conventional official method. The toxic equivalent quantity (TEQ) was calculated using WHO-TEF (1998).

\section{GC/MS measurement}

The dioxins were subjected to HRGC/HRMS with a JEOL JMS-700 mass spectrometer (JEOL Ltd., Tokyo, Japan) equipped with a DB-17HT capillary column $(30 \mathrm{~m} \times 0.25 \mathrm{~mm}$ i.d., $0.15 \mu \mathrm{m}$ film thickness) for PCDD/Fs and non-ortho PCBs, or a DB-5MS capillary column $(30 \mathrm{~m} \times 0.25 \mathrm{~mm}$ i.d., $0.25 \mu \mathrm{m}$ film thickness) for mono-ortho PCBs, with helium as the carrier gas at a linear velocity of $35 \mathrm{~cm} \mathrm{~s}^{-1}$ in the splitless injection mode $(1 \mu \mathrm{L})$. The $\mathrm{GC}$ program was as follows: $150^{\circ} \mathrm{C}(1 \mathrm{~min})$

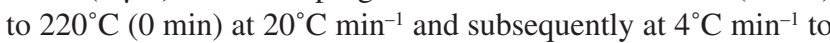
$280^{\circ} \mathrm{C}$, then maintained for $16.5 \mathrm{~min}$ at $280^{\circ} \mathrm{C}$, for both DB$17 \mathrm{HT}$ and DB-5MS columns. The injector temperature was $280^{\circ} \mathrm{C}$ and the $\mathrm{GC} / \mathrm{MS}$ interface temperature was held at $280^{\circ} \mathrm{C}$. The MS was operated in the selected ion-monitoring mode with a mass resolution of 10000 , and the electron impact ionization energy was $38 \mathrm{eV}$ with an ion source temperature of $260^{\circ} \mathrm{C}$. PCDD/Fs, non-ortho PCBs and mono-ortho PCBs were quantified using one molecular $\left(\mathrm{M}^{+}\right)$ion, $(\mathrm{M}+2)^{+}$and $(\mathrm{M}+4)^{+}$ion.

\section{Large-volume injection}

For LVI, a newly developed LVI system, named LaviStoma (AiSTI SCIENCE, Wakayama, Japan), including an SSIL device, which was installed in the back injector port of an 


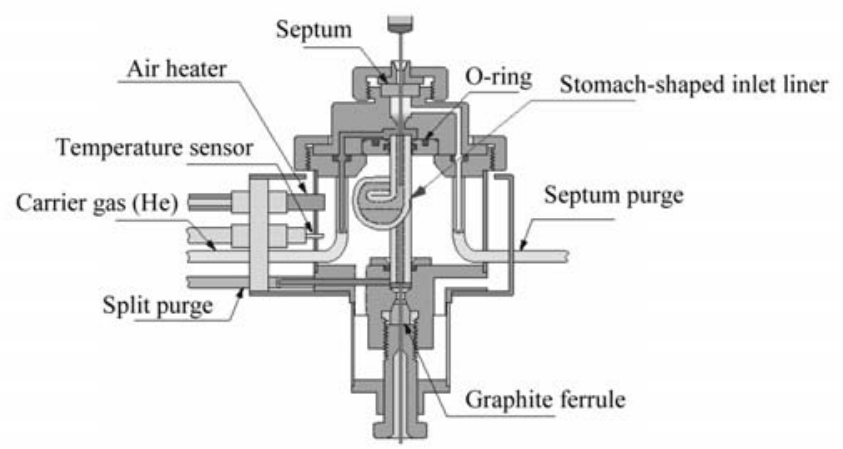

Fig. 1 Inside structure of LaviStoma.

Agilent 6890 GC equipped with a JMS-700 mass spectrometer, was used. The LaviStoma was a kind of injection unit, which was composed of mainly an SSIL, an air heater, a temperature sensor, a carrier gas and a vent line, as shown in Fig. 1. For installation in a GC, a conventional split/splitless injection unit was exchanged for an SSIL device unit. The LaviStoma was applicable for not only the LVI method, but also a conventional split/splitless injection method.

The GC columns used in the analysis for the LVI were the same columns used in the conventional splitless injection. The temperature conditions for LaviStoma and the capillary column were as follows: the initial injection port temperature was increased from $120^{\circ} \mathrm{C}(0.1 \mathrm{~min})$ to $280^{\circ} \mathrm{C}$ at $80^{\circ} \mathrm{C} \mathrm{min}{ }^{-1}$, and then the temperature was maintained for $30 \mathrm{~min}$ at $280^{\circ} \mathrm{C}$. The solvent purge time was set at $6 \mathrm{~s}$. The GC oven temperature program was as follows: $100^{\circ} \mathrm{C}(2 \mathrm{~min})$ to $200^{\circ} \mathrm{C}(0 \mathrm{~min})$ at $20^{\circ} \mathrm{C} \min ^{-1}$ and subsequently at $4^{\circ} \mathrm{C} \min ^{-1}$ to $280^{\circ} \mathrm{C}$, then maintained for $9 \mathrm{~min}$ at $280^{\circ} \mathrm{C}$.

\section{Results and Discussion}

\section{Operating conditions for LVI using LaviStoma}

LaviStoma was developed to overcome the drawbacks of the available LVI methods. Its name was derived from its being uniquely shaped like a stomach, having a large capacity (Figs. 1 and 2) that enables total sample evaporation in the inlet. The device has a temperature range of $40-300^{\circ} \mathrm{C}$ and a programmed rate that reaches as high as $150^{\circ} \mathrm{C} \mathrm{min}^{-1}$. In LaviStoma, high boiling-point solvents, such as toluene or decane, can be used. The principle of LaviStoma is as follows (Fig. 2). First, the injector is kept at a low temperature, and the large-volume of a sample injected into the SSIL remains in the liquid phase in the liner. Then, the solvent is evaporated and the sample is concentrated under carrier gas flow, similar to solvent evaporation by a nitrogen gas stream. Next, the sample is introduced into a capillary column by raising the temperature of the insert in the splitless mode. Finally, any residual solvent or high boiling-point impurities is purged out in the split mode.

The initial temperature and time parameters are critical factors for the operation of LaviStoma. The advisable temperature program of LaviStoma and the column oven in this experiment is shown in Fig. 3. During from the 1st to 2 nd stages of solvent evaporation and sample concentration, the temperature of the injector port is set near to the solvent boiling-point to evaporate the solvent, and the column oven temperature is set at a value lower than the solvent boiling-point to prevent the solvent in the gas phase from flowing into the analytical GC capillary column. In the LaviStoma system, most of the solvent vapor was

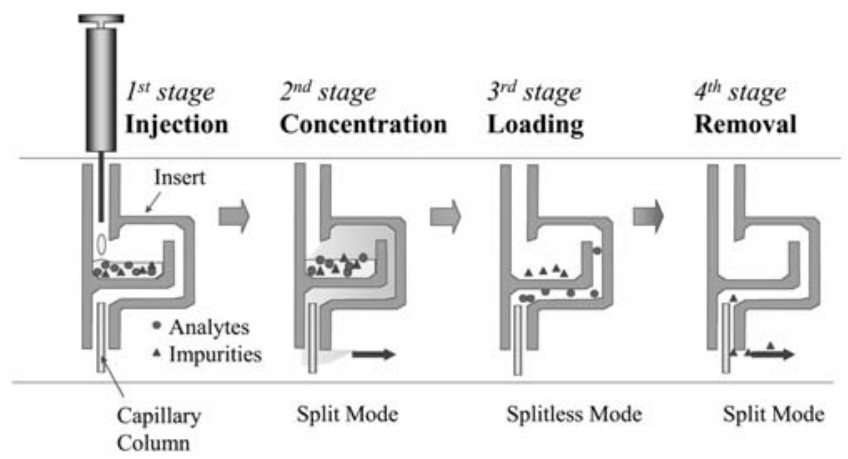

Fig. 2 Principle of PTV injection with LaviStoma. 1st stage: the injector is kept at a low temperature, and the large-volume of sample injected into the SSIL remains in the liquid phase in the liner. 2nd stage: the solvent is evaporated and the sample is concentrated under carrier gas flow. 3rd stage: the sample is introduced into a capillary column by raising the temperature of the SSIL in the splitless mode. 4th stage: residual solvent or high boiling-point impurities are purged out in the split mode.

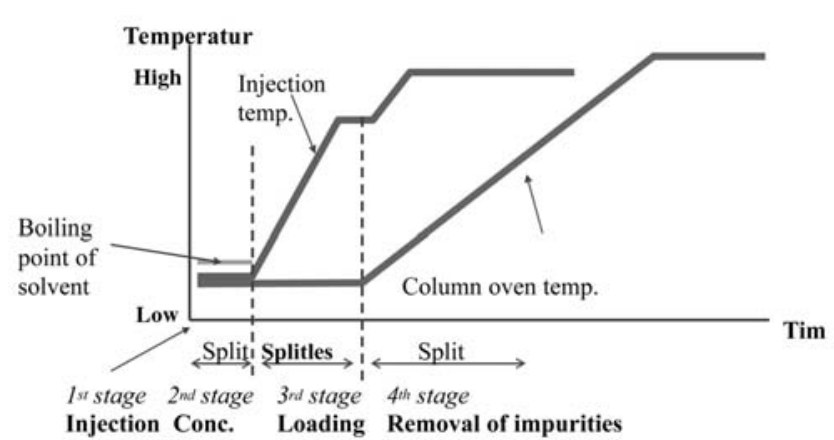

Fig. 3 Advisable temperature program for the operation of LaviStoma. Each stage (1st, 2nd, 3rd and 4th) is coincided at each stage shown in Fig. 1.

excreted via the split vent line. However, the remaining small amount of the vapor might be flowed into the capillary column. When the column temperature is lower than the boiling-point of the solvent, the solvent vapor can be recondensed to the liquid state at the inlet of the column. The solvent in the gas phase seemed to be prevented from flowing into the analytical GC capillary column.

After the solvent has evaporated, which is excluded through a split purge line, the column oven temperature is programmed to separate the analytes. The boiling-point of the solvent is known to depend on the surrounding atmospheric pressure, i.e., the head pressure in the SSIL generated by carrier gas flow. The boiling-point is calculated using the following Antoine equation:

$$
\log (p / \mathrm{mmHg})=A-B /\left(C+\left(t /^{\circ} \mathrm{C}\right)\right)
$$

where $p$ is pressure and $t$ is temperature; $A, B$ and $C$ are constants.

In the LVI method described here, the temperatures of the injector port and the column oven are vital to realizing high recovery and reproducibility. We conducted experiments to determine the optimal temperatures of the injector and the column oven. The injection port temperature (1st and 2 nd stages), which should be below the solvent boiling-point 
(A)

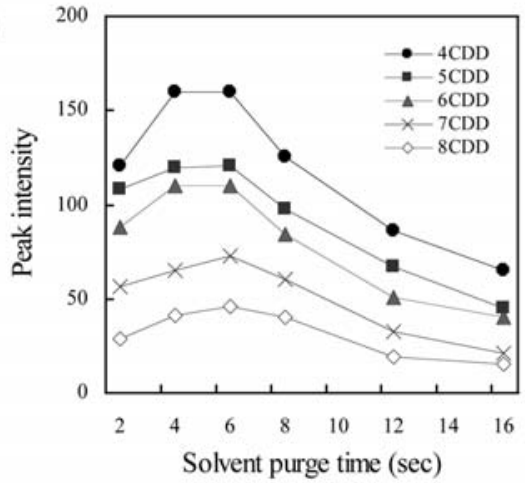

(B)

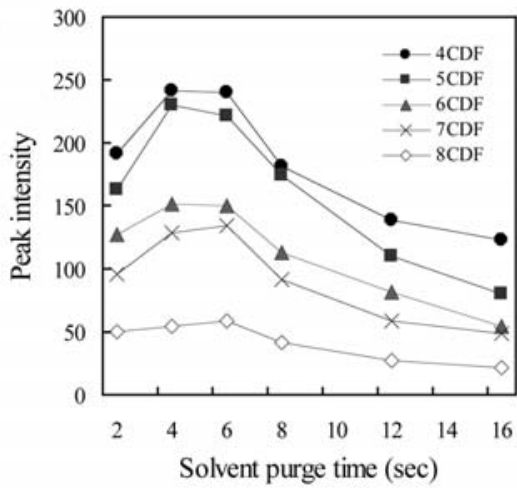

Fig. 4 Effect of the solvent purge time of LaviStoma on the peak intensity of (A) PCDDs and (B) PCDFs. During solvent purge, solvent vapor was excreted via split vent line. 4CDD, 2378-TCDD; 5CDD, 12378-PeCDD; 6CDD, 123678-HxCDD; 7CDD, 1234678-HpCDD; 8CDD, OCDD; 4CDF, 2378-TCDF; 5CDF, 23478-PeCDF; 6CDF, 234678-HxCDF; 7CDF, 1234678-HpCDF; 8CDF, OCDF.

(decane, $174^{\circ} \mathrm{C}$; toluene, $110^{\circ} \mathrm{C}$ under atmospheric pressure) used in this study, was set at $120^{\circ} \mathrm{C}$. Moreover, the initial column oven temperature should be below the injection port temperature.

In the sample analysis, $50 \mu \mathrm{L}$ of the internal standard decane solution for the calibration was added to approximately $1 \mathrm{~mL}$ of a toluene solution to obtain $5 \%$ decane/toluene, after concentration by activated carbon/silica-gel column chromatography at the final cleanup stage. Therefore, the optimal operating conditions for LaviStoma were examined using authentic PCDD/F standards dissolved in 5\% decane/toluene. Figure 4 shows the effect of the solvent purge time of LaviStoma on the peak intensity of PCDD/Fs. The optimum solvent purge time was from 4 to $6 \mathrm{~s}$, as shown in Fig. 4 , when $20 \mu \mathrm{L}$ of the standard solution was injected. We can not correctly explain the critical mechanisms about the results shown in Fig. 4. However, we surmise it to be as follows: A short solvent purge time led to insufficient evaporation of the solvent at the SSIL. Also, the target compounds were not sufficiently introduced (at the 3rd stage) into the capillary column, since the existence of much solvent vapor remaining in the SSIL disturbed it. On the other hand, a long solvent purge time led to co-evaporation of a part of the analytes, eventually resulting in the loss of analytes. We adopted a solvent purge time of $6 \mathrm{~s}$ to ensure that no solvent remained in the SSIL. Although there was 5\% decane in the toluene solution, almost all of the decane was removed together with toluene by evaporation at the solvent purge time, at the 2nd stage of LaviStoma operation.

\section{Linearity and repeatability}

The relationship between the peak area and the injected sample volume was examined when different stepwise volumes $(1,5,10,20 \mu \mathrm{L})$ of the standard solution $\left(2 \mathrm{ng} \mathrm{mL}^{-1} \mathrm{PCDD} / \mathrm{Fs}\right.$ in $5 \%$ decane/toluene, $10 \mathrm{ng} / \mathrm{mL}$ non-ortho $\mathrm{PCBs}$ in $5 \%$ decane/toluene) were injected using LaviStoma under the operating conditions mentioned above. A good linear relationship was found between the peak area and the injected volume from 1 to $20 \mu \mathrm{L}$, although the operating conditions were not always the best for all volume injections, except for $20 \mu \mathrm{L}$ injection. The results suggest that the proposed LVI method demonstrated a 20-times higher sensitivity when $20 \mu \mathrm{L}$ of sample was injected, compared with $1 \mu \mathrm{L}$ sample injection in the conventional splitless injection method.
Table 1 Repeatability of the peak response by multiple large-volume injection

\begin{tabular}{|c|c|c|c|c|c|}
\hline & \multicolumn{5}{|c|}{ Peak area ${ }^{a}$} \\
\hline & \multicolumn{2}{|c|}{ Range } & \multirow{2}{*}{ Average } & \multirow{2}{*}{$\mathrm{SD}$} & \multirow{2}{*}{$\mathrm{RSD}, \%$} \\
\hline & Min & $\operatorname{Max}$ & & & \\
\hline 2378-TCDD & 670 & 696 & 683 & 8.2 & 1.2 \\
\hline 12378-PeCDD & 503 & 552 & 527 & 21.0 & 4.0 \\
\hline 123678-HxCDD & 372 & 411 & 396 & 13.1 & 3.3 \\
\hline 1234678-HpCDD & 237 & 302 & 270 & 28.0 & 10.4 \\
\hline OCDD & 178 & 212 & 190 & 12.7 & 6.7 \\
\hline 2378-TCDF & 1089 & 1189 & 1146 & 41.1 & 3.6 \\
\hline 23478-PeCDF & 878 & 1012 & 951 & 41.4 & 4.4 \\
\hline 234678-HxCDF & 606 & 695 & 643 & 31.6 & 4.9 \\
\hline 1234678-HpCDF & 432 & 543 & 471 & 37.5 & 8.0 \\
\hline OCDF & 201 & 282 & 241 & 31.5 & 13.0 \\
\hline PCB \#77 & 1044 & 1085 & 1063 & 15.0 & 1.4 \\
\hline PCB \#126 & 928 & 981 & 959 & 20.7 & 2.2 \\
\hline PCB \#169 & 725 & 776 & 758 & 16.2 & 2.1 \\
\hline
\end{tabular}

PCDD/Fs: Standard $2 \mathrm{ng} \mathrm{mL} \mathrm{mL}^{-1}$ (5\% decane/toluene), $20 \mu \mathrm{L}$ injection ( $n$ $=7$ ).

Non-ortho PCBs: Standard $10 \mathrm{ng} \mathrm{mL}^{-1}$ (5\% decane/toluene), $20 \mu \mathrm{L}$ injection $(n=7)$.

a. Data are original peak area values of HRGC/HRMS.

In order to confirm the repeatability of the LVI method, $20 \mu \mathrm{L}$ of a standard solution ( $2 \mathrm{ng} \mathrm{mL}^{-1} \mathrm{PCDD} / \mathrm{Fs}$ in $5 \%$ decane/toluene, $10 \mathrm{ng} \mathrm{mL}^{-1}$ non-ortho PCBs in $5 \%$ decane/toluene) was injected 7 times, employing the LVI method with LaviStoma. The relationship between the peak area and each injection is shown in Table 1. While we measured 7 kinds of PCDDs, 10 kinds of PCDFs and 4 kinds of non-ortho PCBs in the experiment, we showed the representative isomer in each homologue of 4CDDs, 5CDDs，6CDDs，7CDDs，8CDD，4CDFs，5CDFs，6CDFs, $7 \mathrm{CDF}$ a and $8 \mathrm{CDF}$, since other isomers in each homologue showed a similar behavior. As a result, most of the peak areas showed nearly constant values, and the RSD values demonstrated high repeatability. The detection limits $\left(\mathrm{pg} \mathrm{g}^{-1}\right)$, which were estimated by the signal-to-noise ratio of each dioxin $\left({ }^{12} \mathrm{C}\right)$ standard for the respective analytes, were as follows: 0.02 for $4-5 \mathrm{CDD} / \mathrm{Fs}, 0.05$ for $6-7 \mathrm{CDD} / \mathrm{Fs}$, and 0.1 for $\mathrm{OCDD} / \mathrm{F}$, non-ortho PCBs and mono-ortho PCBs. These data were not 

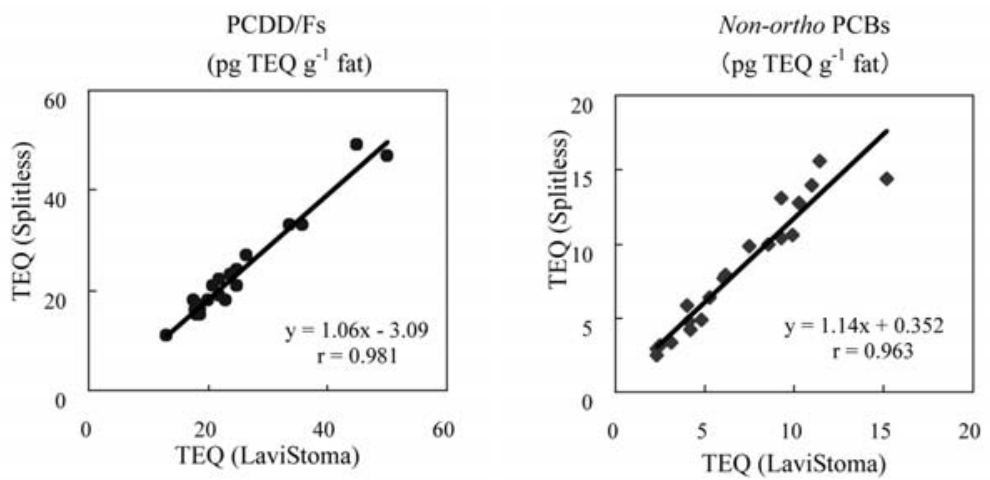

Fig. 5 Correlation of TEQs (PCDD/Fs or non-ortho PCBs) obtained by between splitless injection and the LVI method. The samples were human milk (17 samples) and human plasma (4 samples).

compensated by using surrogates. Therefore, we thought that the small loss of dioxins was caused during the concentration step by using the LaviStoma.

Comparison of net values and TEQ obtained by splitless injection and LaviStoma

In order to confirm the applicability of the LVI method, the correlation of the data obtained by the conventional regularvolume splitless injection of previously concentrated sample with those obtained by the proposed LVI method using LaviStoma was examined. In this experiment, human milk (17 samples) and human plasma (4 samples) were analyzed. Sample preparation was accomplished according to the procedures described in Experimental. Regarding the monoortho PCB analysis, we thought that the analysis does not need LVI, because the residual level is usually higher than that of $\mathrm{PCDD} / \mathrm{Fs}$ or non-ortho PCBs, and there is no need to concentrate them by solvent evaporation by a nitrogen gas stream.

Figure 5 shows a significant correlation $(r>0.97)$ between the LaviStoma values and the conventional splitless injection values for PCDD/Fs and Co-PCBs in human milk $(n=17)$ and plasma $(n=4)$ samples. These data demonstrated that the proposed LVI method is applicable for the determination of dioxins in human milk and plasma. Using the LVI method, micro concentration by solvent evaporation by a nitrogen gas stream at the final stage of sample preparation can be omitted, which has been a problem in the conventional analytical procedure described above. Although the SSIL device also needs a gas stream for removing the solvent, the gas (helium) flow for the SSIL device can be strictly controlled by GC. Also, the gas exhausted was not excreted outside the GC, since the solvent gas could be eliminated via a split outlet. Therefore, the LVI method using the SSIL device (LaviStoma) has other advantages in that it can reduce the sample preparation time by omitting the last concentration step as well as to lessen the danger of the loss of dioxins by volatilization and the exposure of laboratory technicians to dioxins during solvent evaporation by the nitrogen gas stream.

\section{Conclusions}

A $20-\mu \mathrm{L}$ volume of sample solution (5\% decane/toluene) can be injected into the proposed LaviStoma system for the determination of trace amounts of dioxins in human milk and plasma samples. The LaviStoma system with SSIL was able to omit time-consuming solvent evaporation steps; and furthermore, this method might be able to decrease the exposure to dioxins of laboratory technicians who perform solvent evaporation by a nitrogen gas stream by manual operation. The results showed that the proposed LVI method using LaviStoma has a capability of practical use instead of using the conventional splitless injection method. Our proposed method is simpler and easier to use than other LVI methods.

\section{Acknowledgements}

This work was supported in part by Health Sciences Research Grants from the Ministry of Health, Labour and Welfare of Japan.

\section{References}

1. H. G. J. Mol, H.-G. M. Janssen, C. A. Cramers, J. J. Vreuls, and U. A. Th. Brinkman, J. Chromatogr., A, 1995, 703, 277.

2. W. Engeward, J. Teske, and J. Efer, J. Chromatogr., A, 1999, 856,259

3. F. J. Lopez, J. Beltran, M. Forcada, and F. Hernandez, J. Chromatogr., A, 1998, 823, 25.

4. M. Hada, M. Takino, T. Yamagami, S. Daishima, and K. Yamaguchi, J. Chromatogr., A, 2000, 874, 81.

5. A. Aguera, L. Piedra, M. D. Hernando, and A. R. Fernandez-Alba, J. Chromatogr., A, 2000, 889, 261.

6. L. Rosenblum, T. Hieber, and J. Morgan, J. AOAC Int., 2001, 84, 891.

7. E. Korenkova, E. Matisova, and J. Slobodnik, J. Chromatogr. Sci., 2004, 42, 531.

8. F. David, P. Sandra, A. Hoffmann, and J. Gerstel, Chromatographia, 1992, 34, 259.

9. H. Tajima, N. Shimamura, and M. Aoki, Bunseki Kagaku, 1993, 42, 573.

10. K. Urano, M. Kato, Y. Nagayanagi, Y. Saito, A. Aono, J. Nagata, and H. Syudo, Chemosphere, 2001, 43, 425.

11. T. Iida and T. Todaka, Ind. Health, 2003, 41, 197.

12. W. Vogt, K. Jacob, and H. W. Obwexer, J. Chromatogr., 1979, 174, 437

13. K. Grob and M. Biedermann, J. Chromatogr., A, 1996, 750, 11.

14. M. W. J. van Hout, R. A. de Zeeuw, J. P. Franke, and G. J. de Jong, J. Chromatogr., B, 1999, 729, 199. 
15. K. Grob, Jr., G. Karrer, and M.-L. Riekkola, J. Chromatogr., 1985, 334, 129.

16. B. Grolimund, E. Boselli, K. Grob, R. Amad, and G. Lercker, J. High Resolut. Chromatogr., 1998, 21, 378.

17. K. Grob and B. Schilling, J. High Resolut. Chromatogr., Chromatogr. Commun., 1985, 8, 726.

18. A. J. H. Louter, E. Bosma, J. C. A. Schipperen, J. J. Vreuls, and U. A. Th. Brinkman, J. Chromatogr., B, 1997, 689, 35.

19. K. Grob, J. High Resolut. Chromatogr., 1990, 13, 540.

20. K. Grob and S. Brem, J. High Resolut. Chromatogr., 1992, 15,715 .

21. P. Magni and Th. Porzano, J. Sep. Sci., 2003, 26, 1491.

22. M. Herraiz, G. Reglero, E. Loyola, and T. Herraiz, J. High Resolut. Chromatogr., 1987, 10, 598.

23. Y. Saito, S. Kodama, A. Matsunaga, and A. Yamamoto, $J$. AOAC Int., 2004, 87, 1356.

24. S. de Koning, M. Kurano, H.-G. Janssen, and U. A. Th.
Brinkman, J. Chromatogr., A, 2004, 1023, 165.

25. H. M. Müller and H.-J. Stan, J. High Resolut. Chromatogr., 1990, $13,759$.

26. H. G. J. Mol, P. J. M. Hendriks, H.-G. Janssen, C. A. Cramers, and U. A. Th. Brinkman, J. High Resolut. Chromatogr., 1995, 18, 124.

27. K. Grob, E. Boselli, and G. Lercker, J. High Resolut. Chromatogr., 1999, 22, 149.

28. T. Hankemeier, S. J. Kok, R. J. J. Vreuls, and U. A. Th. Brinkman, J. Chromatogr., A, 1999, 841, 75.

29. K. Saito, M. Takekuma, M. Ogawa, S. Kobayashi, Y. Sugawara, M. Ishizuka, H. Nakazawa, and Y. Matsuki, Bull. Environ. Contam. Toxicol., 2003, 70, 636.

30. K. Saito, M. Takekuma, M. Ogawa, S. Kobayashi, Y. Sugawara, M. Ishizuka, H. Nakazawa, and Y. Matsuki, Chemosphere, 2003, 53, 137. 